Methods: We used the Parent/Caregiver form in order to assess psychometric characteristics of the VABS-II and to develop norms for the French population. VABS-II comprises 4 domains, 11 subdomains, and an optional maladaptive behavior index. The French translation of the VABS-II followed standard cross-cultural translation methods. The study was performed in the Rhône-Alpes-Auvergne department comprising $12 \%$ of France inhabitants and well representing the French general population.

Results: From 4576 VABS-II questionnaires distributed, 1707 were returned and 1654 were analyzed. The reason for exclusion was the impossibility to score one of the subdomains. From 174 questionnaires included in the test-retest, 95 were analyzed, and 79 questionnaires were excluded because 86 under 34 days, $8>3$ months, and one participant that changed age group between test and retest. Scores based on French norms fluctuated around values based on US norms on all subdomains.

Conclusions: The French Vineland questionnaire is the single test with the adequate norms to allow identifying children with adaptive behavior difficulties. It should be used as a complement of the assessment of the intellectual quotient, according to DSM V, for the diagnosis of intellectual disabilities. It must be done in reference to the developmental and cultural standards specific to the environment in which the person is evolving.

Disclosure: No significant relationships.

Keywords: autism spectrum disorder; French norms; adaptative behavior; validation

\section{Schizophrenia and other psychotic disorders}

\section{8}

\section{Drug attitude inventory is relevant to LAI treatment persistence in schizophrenia: Preliminary results}

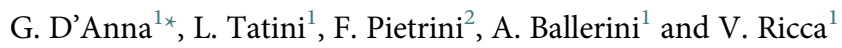

${ }^{1}$ Department Of Health Sciences, University of Florence, Florence, Italy and ${ }^{2}$ Department Of Mental Health And Addictions, Central Tuscany NHS Trust, Florence, Italy

${ }^{*}$ Corresponding author.

doi: 10.1192/j.eurpsy.2021.422

Introduction: Patients' attitudes and subjective experience are crucial in the management of severe mental illness, but their practical value is overlooked.

Objectives: To identify predictors of future adherence to LAI antipsychotic maintenance treatment of schizophrenia among socio-demographic, clinical, and psychometric characteristics including Drug Attitude Inventory-10 (DAI-10) and Subjective Well-being under Neuroleptics short form (SWN-K) scores.

Methods: Retrospective baseline data from 53 clinically stable outpatients with schizophrenia switched from oral to LAI therapy were collected. Patients continuing treatment at the time of analysis $(n=29)$ were compared to those who had discontinued it $(n=24)$. Selected variables were further evaluated in survival analyses.

\begin{tabular}{llll}
\hline & $\begin{array}{c}\text { Continued } \\
\text { treatment }\end{array}$ & $\begin{array}{c}\text { Discontinued } \\
\text { treatment }\end{array}$ & $\chi^{2}$ or t \\
\hline $\begin{array}{l}\text { Treatment persistence } \\
\text { (months) }\end{array}$ & $63.79 \pm 21.01$ & $23.88 \pm 25.80$ & $6.21^{\star \star}$ \\
\hline Age (years) & $39.17 \pm 10.11$ & $35.58 \pm 13.39$ & 1.11 \\
\hline Male & $15(51.7 \%)$ & $13(54.1 \%)$ & 0.03 \\
\hline Single & $20(69.0 \%)$ & $15(62.5 \%)$ & 0.25 \\
\hline Instruction (years) & $13.28 \pm 3.31$ & $11.83 \pm 3.56$ & 1.53 \\
\hline Employed & $20(69.0 \%)$ & $7(29.2 \%)$ & $8.32^{\star \star}$ \\
\hline Illness duration (years) & $17.69 \pm 10.53$ & $13.42 \pm 11.36$ & 1.42 \\
\hline Previous hospitalisations & $2.10 \pm 1.32$ & $2.67 \pm 1.86$ & -1.29 \\
\hline MADRS & $13.59 \pm 9.06$ & $14.67 \pm 8.99$ & -0.43 \\
\hline YMRS & $5.52 \pm 5.57$ & $6.00 \pm 9.94$ & -0.22 \\
\hline p-PANSS & $12.17 \pm 5.20$ & $14.38 \pm 6.13$ & -1.41 \\
\hline n-PANSS & $10.90 \pm 5.39$ & $15.63 \pm 7.93$ & $-2.48^{\star}$ \\
\hline g-PANSS & $29.38 \pm 10.33$ & $33.63 \pm 10.26$ & -1.49 \\
\hline PANSS & $52.66 \pm 17.57$ & $63.96 \pm 20.61$ & $-2.15^{\star}$ \\
\hline DAI-10 & $3.86 \pm 4.96$ & $-1.13 \pm 5.80$ & $3.38^{\star \star}$ \\
\hline SWN-K & $74.93 \pm 23.07$ & $81.00 \pm 15.60$ & -1.09 \\
\hline & & & \\
\hline
\end{tabular}

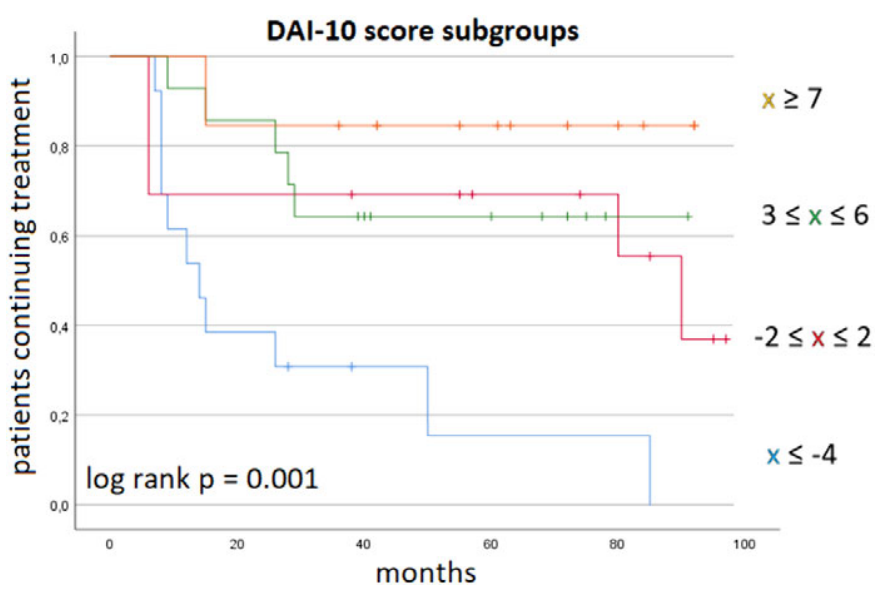

Results: Between-group differences are presented in Table $1{ }^{* *}$ : $\left.\mathrm{p}<0.01{ }^{*}: \mathrm{p}<0.05\right)$.

Cox regression analysis included instruction, employment, hospitalisations, PANSS subscales and DAI-10 scores: a protective role against treatment discontinuation was outlined only for employment (HR 0.16; 95\%CI 0.05-0.50) and higher DAI-10 scores (HR 0.85; 95\%CI 0.78-0.94). DAI-10 scores delineated distinct adherence trajectories (Figure 1).

Conclusions: Baseline DAI-10 scores may identify patients at risk of dropout after switching to LAI.

Disclosure: No significant relationships.

Keywords: Drug Attitude Inventory; adherence; schizophrénia; LAI 
0249

\section{Prediction of quality of life in schizophrenia using machine learning models on data from clinical antipsychotic trials of intervention effectiveness (CATIE) schizophrenia trial}

\author{
M. Beaudoin ${ }^{1,2,3 *}$, S. Potvin ${ }^{1,2}$, A. Hudon ${ }^{1,2}$, C.-E. Giguère ${ }^{2}$ and \\ A. Dumais ${ }^{1,2,4}$ \\ ${ }^{1}$ Psychiatry And Addictology, University of Montreal, Montreal, \\ Canada; ${ }^{2}$ Centre De Recherche, Institut universitaire en santé mentale \\ de Montréal, Montréal, Canada; ${ }^{3}$ Faculty Of Medicine, McGill \\ University, Montreal, Canada and ${ }^{4}$ Psychiatry, Institut national de \\ psychiatrie légale Philippe-Pinel, Montreal, Canada \\ ${ }^{*}$ Corresponding author. \\ doi: 10.1192/j.eurpsy.2021.423
}

Introduction: Schizophrenia is a chronic and severe mental disorder. While research focus remains mainly on negative outcomes, it is questionable whether we are placing enough emphasis on improving their sense of well-being and functioning. This could be accessed through the study of the quality of life (QoL). To date, QoL prediction models mainly focused on neurocognition and psychotic symptoms, but their predictive power remained limited. Objectives: The aim is to accurately predict the QoL within schizophrenia using unsupervised learning methods.

Methods: We computed variables from 952 patients from the CATIE study, a randomized, double-blind clinical trial for schizophrenia treatment. QoL was measured using the HeinrichsCarpenter Quality of Life Scale and potential predictors included almost all available variables: symptoms, neurocognition, medication adherence, insight, adverse effects, etc. By optimizing parameters to reach optimal models, three linear regressions were calculated: (1) baseline predictors of 12-month QoL, (2) 6-month predictors of 12-month QoL, and (3) baseline predictors of 6-month QoL. Adjustments were made to ensure that included variables were not collinear nor redundant with QoL.

Results: Calculated models had adjusted R-squared of 0.918, 0.922 and 0.913 , respectively. Best predictors were medication side effects, sociodemographic and neurocognitive variables. Low psychotic and depressive symptoms were also included, as well as lab values suggesting the absence of problems with chloremia and calcemia.

Conclusions: Calculated predictive models explain almost all subsequent QoL. It appears that physical health variables, generally omitted from mental health-related studies, have an important impact on patients' QoL. Therefore, interventions should also consider these aspects.

Disclosure: No significant relationships.

Keywords: schizophrénia; quality of life; machine learning; predictive models

\section{0}

Efficacy and tolerability of lurasidone in schizophrenia: A systematic review and meta-analysis of short-term, randomized, placebo controlled trials

K. Hagi ${ }^{1 \star}$, T. Nosaka ${ }^{1}$, J. Kane ${ }^{2}$ and C. Correll ${ }^{3}$

${ }^{1}$ Medical Affairs, Sumitomo Dainippon Pharma Co., Ltd., Tokyo, Japan; ${ }^{2}$ Psychiatry Research, The Zucker Hillside Hospital, Glen Oaks,
United States of America and ${ }^{3}$ Department Of Child And Adolescent Psychiatry, Psychosomatic Medicine And Psychotherapy, Charité University Medical Center, Berlin, Charite Berlin, Padua, Germany ${ }^{\star}$ Corresponding author. doi: 10.1192/j.eurpsy.2021.424

Introduction: Lurasidone is an atypical antipsychotic approved for the treatment of patients with schizophrenia. We report on a metaanalysis focusing on both the efficacy and safety/tolerability of lurasidone in the treatment of patients with schizophrenia.

Objectives: To obtain pooled estimates from placebo-controlled clinical trials on the efficacy and safety/tolerability of lurasidone in schizophrenia.

Methods: We selected acute, randomized placebo-controlled trials of lurasidone for schizophrenia. Primary outcome for efficacy was the Positive and Negative Syndrome Scale (PANSS) change and for "acceptability" was all-cause discontinuation. Secondary outcomes included specific adverse events, body weight change, $\geq 7 \%$ weight gain, and glucose and lipid parameter change.

Results: Across 10 RCTs $(n=3,963$, age $=40.5 \pm 2.3$ years, males $=64.7$ $\%$, trial duration $=6.0$ weeks), lurasidone outperformed placebo regarding the PANSS total score $(\mathrm{N}=10, \mathrm{n}=3,354, \mathrm{SMD}=-0.34,95 \%$ CI: $-0.47--0.21, \mathrm{p}<0.001)$. Stratifying the analysis by dose, lurasidone significantly outperformed placebo at doses 40-160 mg/day. Lurasidone was associated with significantly lower all-cause discontinuation than placebo $(\mathrm{N}=10, \mathrm{n}=3,410, \mathrm{RR}=0.87,95 \% \mathrm{CI}$ : $0.78-0.97, \mathrm{p}=0.014)$. Lurasidone had significantly higher body weight change compared with placebo $(\mathrm{N}=10, \mathrm{n}=3,359, \mathrm{SMD}=0.17,95 \% \mathrm{CI}: 0.09-0.24$, $\mathrm{p}<0.001$ ), but without significant differences regarding $\geq 7 \%$ body weight gain $(\mathrm{N}=9, \mathrm{n}=3,186, \mathrm{p}=0.112)$. Lurasidone did not differ from placebo in total cholesterol $(\mathrm{N}=10, \mathrm{n}=3,140, \mathrm{p}=0.439)$, LDLcholesterol $(\mathrm{N}=7, \mathrm{n}=2,414, \mathrm{p}=0.849)$, triglycerides $(\mathrm{N}=10, \mathrm{n}=3,140$, $\mathrm{p}=0.238)$, and fasting glucose change $(\mathrm{N}=10, \mathrm{n}=3,112, \mathrm{p}=0.633)$.

Conclusions: In short-term trials, lurasidone was efficacious, acceptable and safe, having minimal effect on body weight gain and glucose and lipid metabolism.

Disclosure: K. Hagi is a full time employee of Sumitomo Dainippon Phrma Co., Ltd.

Keywords: lurasidone; meta-analysis; RCT; schizophrénia

\section{O251}

\section{Schizophrenia hospitalizations - a big data approach}

M. Gonçalves-Pinho ${ }^{1 \star}$, J.P. Ribeiro ${ }^{1}$ and A. Freitas ${ }^{2}$

${ }^{1}$ Department Of Psychiatry And Mental Health, Centro Hospitalar do Tâmega e Sousa, Penafiel, Portugal, Penafiel, Portugal and ${ }^{2} 2 \mathrm{~d} 4 \mathrm{~h}$, Center for Health Technology and Services Research (CINTESIS), Porto, Portugal

${ }^{\star}$ Corresponding author.

doi: 10.1192/j.eurpsy.2021.425

Introduction: Schizophrenia is characterized by long hospitalizations and a recurrent use of chronic and acute psychiatric care.

Objectives: The aim of this study was to analyze schizophrenia related hospitalizations in Portugal.

Methods: A retrospective observational study was conducted using a nationwide hospitalization database containing all hospitalizations registered in Portuguese public hospitals from 2008 to 2015. 